\title{
Role of the lipoxin A4 receptor in the development of neutrophil extracellular traps in Leishmania infantum infection
}

\author{
Furong Wei ${ }^{1,2,3,4,5}$, Wenci Gong ${ }^{1,2,3,4,5}$, Junyun Wang ${ }^{1,2,3,4,5}$, Yuetao Yang ${ }^{1,2,3,4,5}$, Jianxiu Liu 1,2,3,4,5, \\ Yanjuan Wang ${ }^{1,2,3,4,5}$ and Jianping Cao ${ }^{1,2,3,4,5^{*}}$
}

\begin{abstract}
Background: Neutrophils play an immunomodulatory role through the release of neutrophil extracellular traps (NETs). NETs are released in response to Leishmania infection, but the mechanism of NET extrusion has not been elucidated. The lipoxin A4 receptor on neutrophils is crucial for the inflammatory response and immune regulation of many diseases, including Leishmania infection. Therefore, in the present study, we tried to explore whether Leishmania infantum promastigotes stimulate neutrophil activation and NET release via activating the lipoxin A4 receptor.

Results: Leishmania infantum promastigotes stimulated neutrophil activity, but blocking of the lipoxin A4 receptor with its antagonist Boc prior to L. infantum stimulation abrogated these effects. Neutrophils showed citrullinated histone $\mathrm{H} 3$ expression and simultaneous NET extrusion on L. infantum stimulation, but a decline in both was observed on blocking of the lipoxin A4 receptor. Moreover, differentiated HL-60 cells with lipoxin A4 receptor silencing showed a decrease in citrullinated histone $\mathrm{H} 3$ expression as compared to the unsilenced $\mathrm{HL}-60$ samples on stimulation with promastigotes.
\end{abstract}

Conclusions: Leishmania infantum promastigotes altered the characteristics of neutrophils and induced NET extrusion by activating the lipoxin A4 receptor. The lipoxin A4 receptor may have potential as a therapeutic target in relation to NET extrusion in the treatment of leishmaniasis, but its mechanisms of action need to be explored in more depth.

Keywords: Lipoxin A4 receptor, Leishmania infantum, Citrullinated histone $\mathrm{H} 3$, Neutrophil extracellular traps

\section{Background}

Leishmaniasis, a vector-borne disease caused by a protozoan flagellate of the genus Leishmania and transmitted by female phlebotomine sand flies, causes a group of diseases which have symptoms that differ according to the causative Leishmania species and the host's ability to develop and control immune responses [1]. The adaptive immune response is known to be critical for resolving Leishmania infection, but innate immune mechanisms have been receiving much attention in the context of

\footnotetext{
*Correspondence: caojp@yahoo.com

${ }^{1}$ National Institute of Parasitic Diseases, Chinese Center for Disease

Control and Prevention, Shanghai 200025, China

Full list of author information is available at the end of the article
}

this disease, especially the activity of neutrophils as antimicrobial effector molecules [2-4].

Neutrophils, as the largest group of immune cells, recruited by numerous parasite-, host- and sand flyderived chemotactic factors in the process [4], are the first to reach the Leishmania infiltration site [5-8] and internalize the parasite $[7,9]$. The internalization process is tightly regulated through three major strategies: phagocytosis, degranulation and the release of neutrophil extracellular traps (NETs). The process of NET release involves the decondensation of chromatin and the disruption of nuclear and granular architecture to allow the mixing of chromatin and antimicrobial granular content in the cytoplasm; this subsequently leads to the release of the chromatin network with anchored granular proteins, 
such as elastase and myeloperoxidase (MPO), into the extracellular space [10]. This novel type of cell program is called NETosis, and NETs are primarily comprised of DNA backbone fibers, antibacterial proteins/peptides, granule proteins, etc.

Neutrophils release NETs in response to L. amazonensis, $L$. infantum, L. major, $L$. donovani and $L$. mexicana infection [9, 11-13]. Some Leishmania species can be captured and killed by NETs [11], while some are protected from NET-mediated killing by lipophosphoglycan [13], the enzyme 3'-nucleotidase/nuclease [12] or by other factors that have yet to be identified [9]. Although many aspects of the immune response to Leishmania species have been unveiled, little is known in terms of the mechanisms of activation of NETosis and the role of NETs.

Leishmania promastigotes, by releasing a chemokine called Leishmania chemotactic factor, can recruit and activate neutrophils via the lipoxin A4 receptor [14]. The lipoxin A4 receptor is crucial for inflammatory response and immune regulation in many diseases [15-17]. In particular, many studies have focused on the role of the lipoxin A4 receptor in neutrophil activation [18]. It has been demonstrated that, in addition to regulation of neutrophil activation and recruitment, this receptor has a profound influence on neutrophil survival and apoptosis with contrasting effects, by mediating aggravation or resolution of the inflammatory response [19]. In particular, anti-inflammatory lipoxin A4 receptor signaling induced by Leishmania promastigotes has been found to increase the phagocytotic ability and enable intracellular survival of Leishmania parasites [20]. Given these complex roles of the lipoxin A4 receptor in neutrophil activity, we were interested in investigating whether Leishmania promastigotes induce NETosis by activating the lipoxin A4 receptor. This study is based on the hypothesis that the lipoxin A4 receptor plays an important role in determining neutrophil fate following neutrophil activation via mediating the production on NETs. This receptor may therefore provide a new pharmacological target in the process of NETosis that occurs during parasitic infections.

\section{Results}

\section{Leishmania infantum promastigotes induce the release} of NETs and are trapped by NETs

To confirm the potential of $L$. infantum to induce the formation of NETs, we cocultured $L$. infantum promastigotes with neutrophils for $5 \mathrm{~h}$. The neutrophils lost their typical rounded morphology and released NETs, which appeared as filamentous structures under a scanning electron microscope (Fig. 1a). Interestingly, the L. infantum promastigotes were found to be entrapped in the NET fibers (Fig. 1b).

\section{The lipoxin A4 receptor is associated with the expression} of citrullinated histone $\mathrm{H} 3$ during $L$. infantum stimulation Given the prominent role of the lipoxin A4 receptor in the anti-inflammatory response of neutrophils, we explored its possible role in the release of citrullinated histone H3 (H3Cit, a significant biomarker of NETs) by using flow cytometric analysis. The thresholds were determined based on the fluorescence levels of the isotype controls. The neutrophils that were positive for H3Cit were counted. As shown in Fig. 2, there were significantly differences among experimental groups
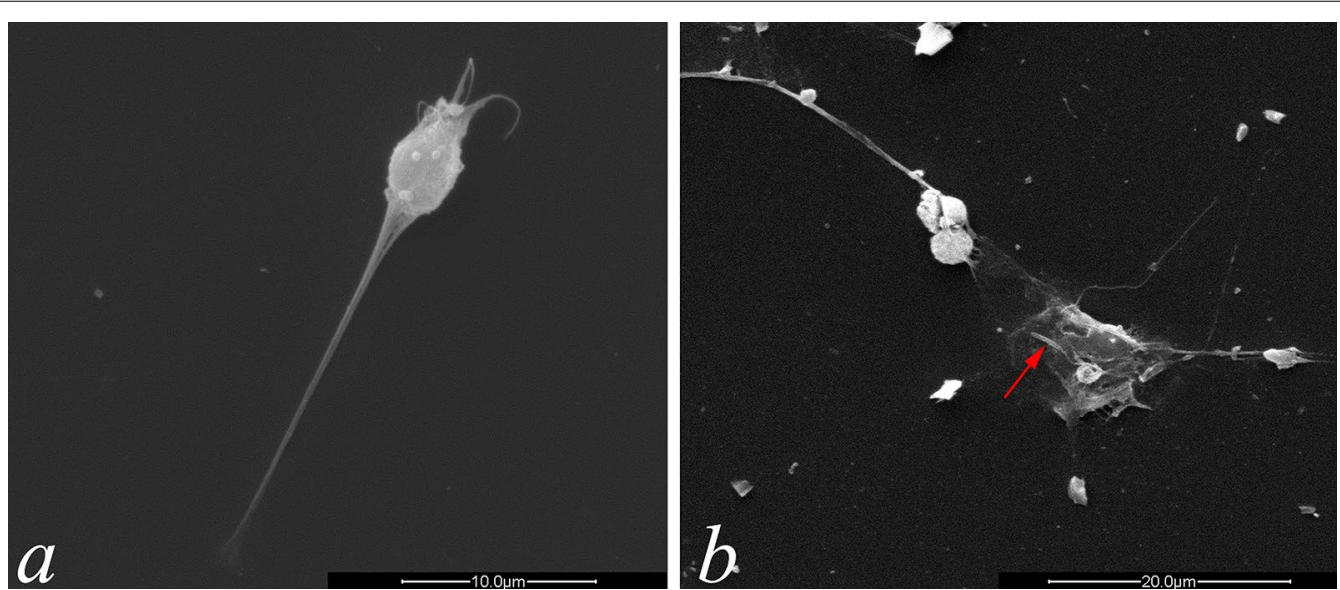

Fig. 1 Scanning electron microscope images of the neutrophils stimulated by L. infantum promastigotes for 5 h. Purified neutrophils were exposed to L. infantum promastigotes for $5 \mathrm{~h}$. The coverslips seeded with samples were fixed with $1 \mathrm{ml}$ of Monti-Graziadei solution and processed for scanning electron microscopy to visualize NET release. a NET structure of a single neutrophil. b NET threads within which a promastigote is trapped (red arrow). Scale-bars: $\mathbf{a}, 10 \mu \mathrm{m} ; \mathbf{b}, 20 \mu \mathrm{m}$ 


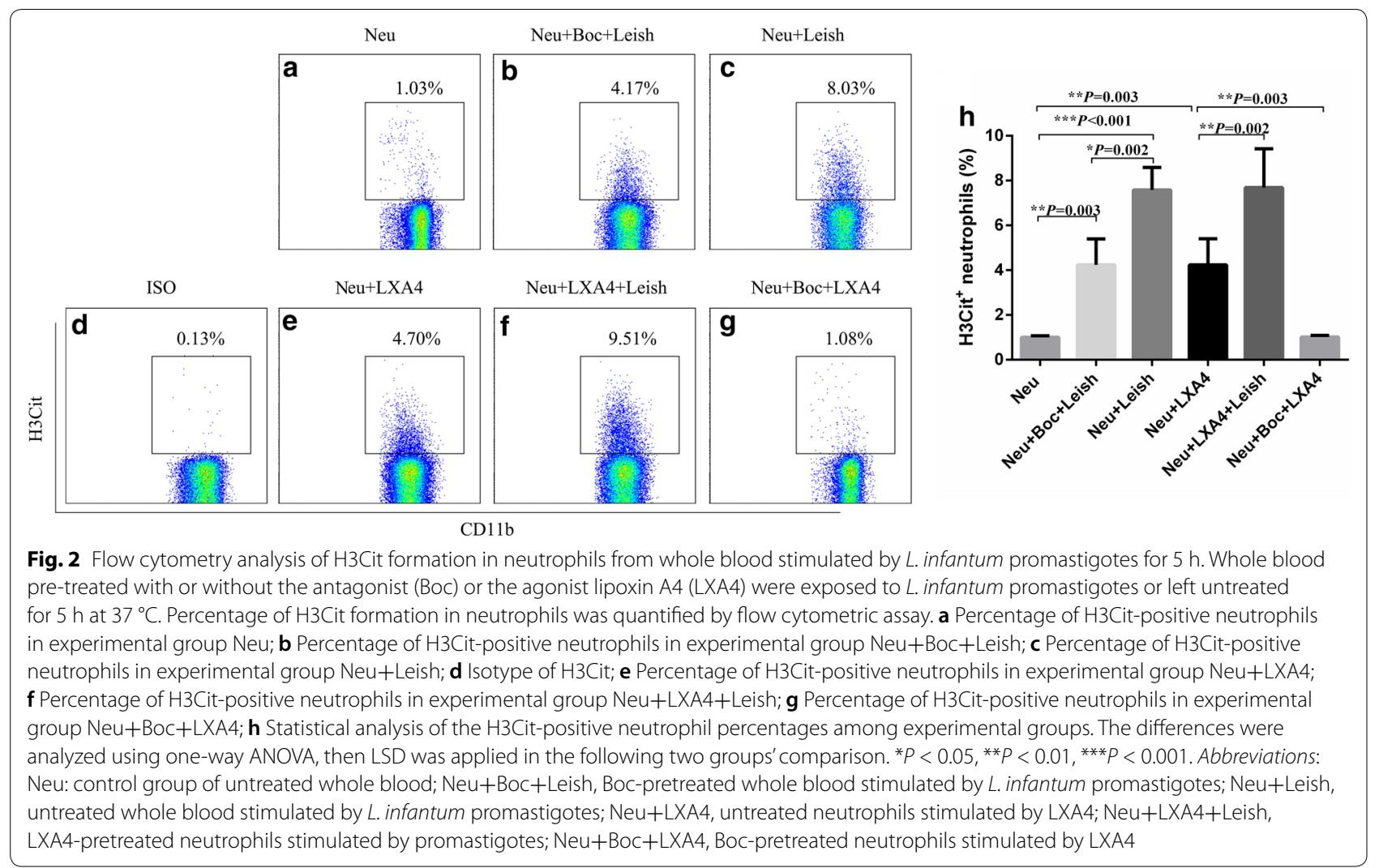

$\left(F_{(5,12)}=2.285, P<0.001\right)$. The proportion of $\mathrm{H} \mathrm{Cit}^{+}$ neutrophils in the Boc-primed whole blood samples as compared to the unprimed whole blood samples on stimulation with promastigotes was significantly decreased ( $P=0.002$ ) (Fig. 2b, c). Despite being less effective than the promastigotes, lipoxin A4 was found to increase $\mathrm{H} 3 \mathrm{Cit}$ extrusion significantly as compared to control group ( $P=0.003$ ) (Fig. 2a, e). Furthermore, Boc was found to reverse the agonistic effects of LXA4 $(P=0.003)$ (Fig. 2e, g).

As neutrophils have a short lifespan after isolation, HL-60 cells that can differentiate into neutrophil-like cells in response to $1.25 \%$ DMSO were used as a model [21] to investigate the impact of the lipoxin A4 receptor on NET expression. We induced the differentiation of HL-60 cells into neutrophil-like cells and the silencing of the lipoxin A4 receptor in neutrophil-like HL-60 cells $\left(t_{(4)}=15.378, P<0.001\right)$ (Fig. 3f), and then determined Leishmania-induced H3Cit production by flow cytometric analysis in both the silenced and non-silenced cells. As shown in Fig. 3, there were significantly differences among experimental groups $\left(F_{(2,6)}=3.264, P<0.001\right)$. The proportion of $\mathrm{H}_{3} \mathrm{Cit}^{+}$neutrophils in the lipoxin A4 receptor-silenced HL-60 samples as compared to the unsilenced HL-60 samples stimulated by promastigotes was significantly decreased $(P<0.001)$ (Fig. 3c, d). Our data thus suggest that the lipoxin A4 receptor mediates Leishmania-associated NETosis.

\section{The lipoxin A4 receptor regulates the release of NETs during $L$. infantum stimulation}

As a significant biomarker of NETs, the antagonistic effect of Boc on H3Cit production implies that the lipoxin A4 receptor may have an effect on NET extrusion. Purified neutrophils primed by an antagonist or agonist were stimulated with promastigotes. Our extrapolation was confirmed by the dsDNA evaluation (Fig. 4) and fluorescence microscopy findings (Fig. 5).

As shown in Fig. 4, there were significantly differences among experimental groups $\left(F_{(5,12)}=1.919, P<0.001\right)$. The dsDNA concentration in the Boc-primed neutrophil samples as compared to the unprimed neutrophil samples stimulated by promastigotes was significantly decreased $(P<0.001)$, and LXA4 exhibited an agonistic effect on the dsDNA concentration as compared to the control samples $(P<0.001)$.

Similarly, the fluorescence microscopy results also showed that NETs positive for DNA, H3Cit and MPO were visible in Boc-untreated and lipoxin A4 pre-treated neutrophils stimulated by promastigotes (Fig. 5c, e). However, most cells in the Boc-primed neutrophil samples showed intact and smaller nuclei (Fig. 5b, f), which 

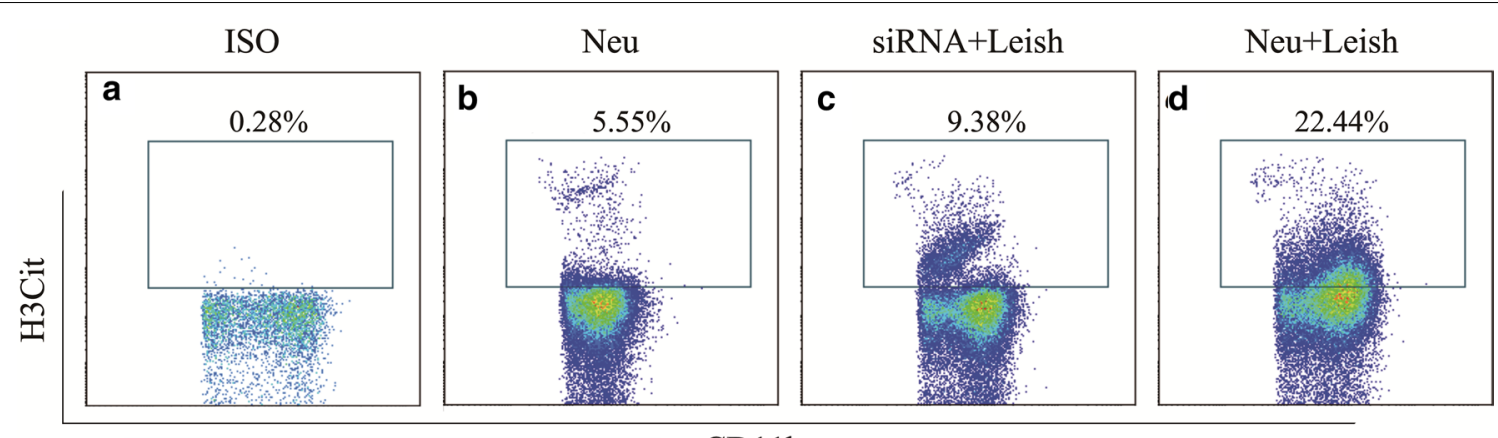

CD11b
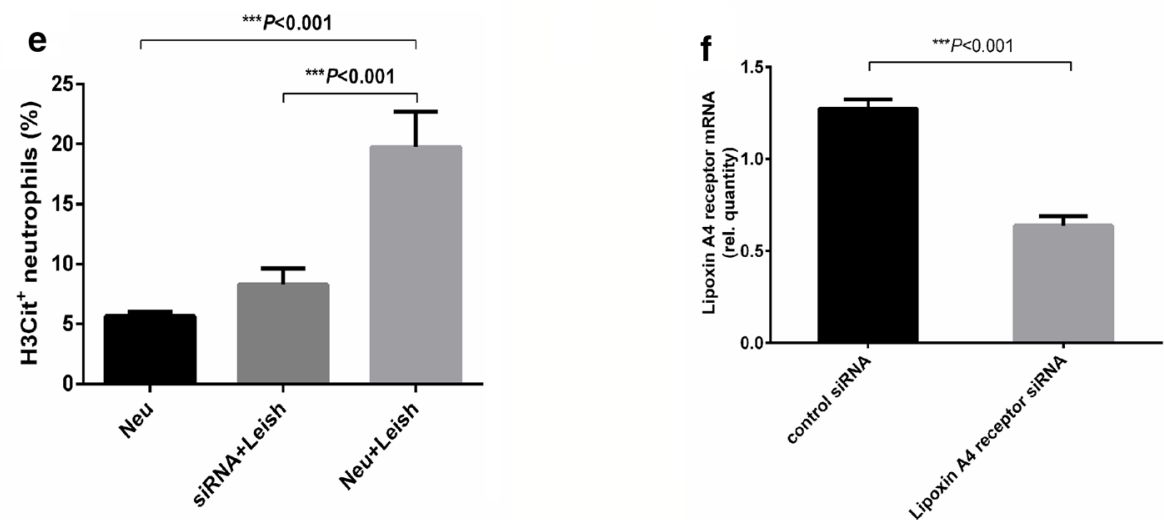

Fig. $3 \mathrm{H} 3$ Cit formation in neutrophil-like HL-60 cells with/without lipoxin A4 receptor silencing after stimulation by L. infantum promastigotes for $5 \mathrm{~h}$. HL-60 cells induced to differentiate into neutrophil-like cells (with 1.25\% DMSO) with or without lipoxin A4 receptor gene silencing were exposed to $\mathrm{L}$. infantum promastigotes or left untreated for $5 \mathrm{~h}$ at $37^{\circ} \mathrm{C}$. H3Cit formation was quantified by flow cytometric assay. a Isotype of H3Cit. b Percentage of H3Cit-positive differentiated $\mathrm{HL}-60$ cells in experimental group Neu; c Percentage of H3Cit-positive differentiated HL-60 cells in experimental group siRNA+Leish; d Percentage of H3Cit-positive differentiated HL-60 cells in experimental group Neu+Leish; e Comparison of the H3Cit-positive neutrophil percentages among experimental groups. The differences were analyzed by one-way ANOVA, then LSD was applied in the following two groups' comparison; $\mathbf{f} \mathrm{HL}-60$ cells were transfected with control siRNA or lipoxin A4 receptor-specific siRNA. Lipoxin A4 receptor transcripts were quantified relative to GAPDH mRNA, using qRT-PCR. Differences were analyzed by Student's $t$-test. ${ }^{*} P<0.05$, ${ }^{* *} P<0.01$, ${ }^{* * *} P<$ 0.001. Abbreviations: Neu, differentiated HL-60 cells without treatment; siRNA+Leish, differentiated HL-60 cells in lipoxin A4 receptor-silenced HL-60 cells exposed to L. infantum promastigotes; Neu+Leish, differentiated HL-60 cells stimulated by L. infantum promastigotes

means that Boc had an inhibitory effect on NET extrusion via its antagonistic effect on the lipoxin A4 receptor.

\section{Discussion}

In the present study, we tried to explore whether the lipoxin A4 receptor plays a role in L. infantum-induced NETs formation. Our findings indicate that NETs positive for DNA, H3Cit and MPO were visible in Leishmaniainfected neutrophils purified from mice. However, most of the lipoxin A4 receptor-blocked neutrophils showed intact and smaller nuclear morphology without MPO fluorescence. Moreover, Boc can reverse the effect of the agonist and downregulate NET extrusion. These observations were quantitatively confirmed by dsDNA measurement. Our research explored the possible role of the lipoxin A4 receptor as a trigger for the release of NETs, and findings confirmed the possibility of such a function. A more important finding is that Leishmania stimulation can significantly increase the NET extrusion of lipoxin A4-primed neutrophils, even though lipoxin A4 could stimulate the NET extrusion. This finding suggest that the lipoxin A4 receptor is one of the receptors that mediates $L$. infantum-induced NET formation, which may rely on a complex regulatory process.

H3Cit plays a crucial role in immune cell chromatin decondensation, as a result of which it can be detected in the nuclei of neutrophils upon stimulation [22, 23]; furthermore, $\mathrm{H} 3 \mathrm{Cit}$ has been proposed to be a central marker of NETs [24]. As flow cytometric assays for direct quantification of NET markers of whole blood [25] can eliminate the effect of isolation procedures on neutrophils, whole blood was used to study the role of the lipoxin A4 receptor in the production of NETs by flow cytometry analysis of specific cell populations without purification. Our research found that the quantity of neutrophils positive for $\mathrm{H} 3 \mathrm{Cit}$ is synchronously decreased 


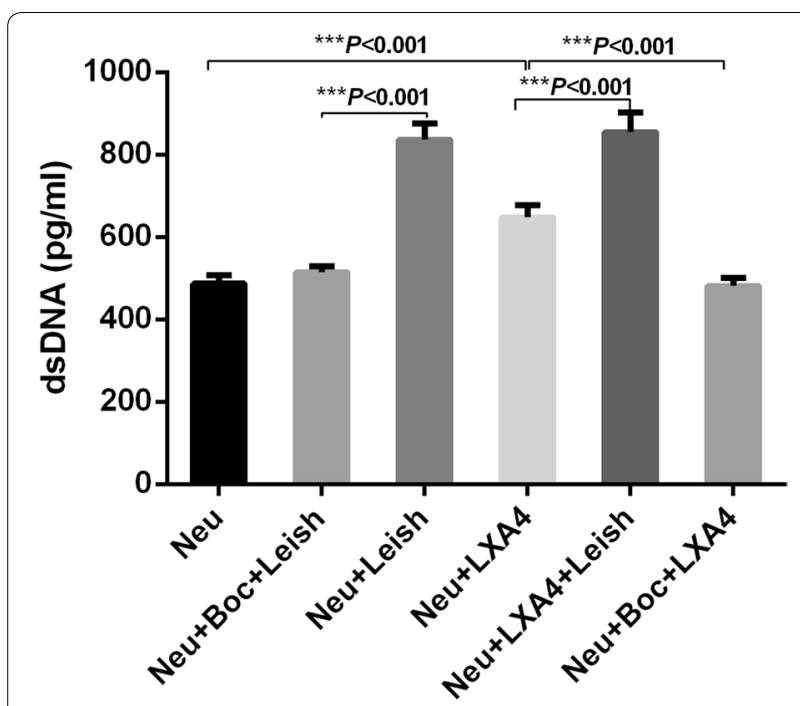

Fig. 4 Comparison of extracellular dsDNA among the experimental groups. Purified neutrophils pre-treated with or without the antagonist (Boc) or the agonist (LXA4) were exposed to L. infantum promastigotes for $5 \mathrm{~h}$ at $37^{\circ} \mathrm{C}$. Extracellular dsDNA in the culture supernatant of the experimental groups were quantified and compared to assess the NETs production. The differences were analyzed using one-way ANOVA, then LSD was applied in the following two groups' comparison. ${ }^{*} P<0.05$, ${ }^{* *} P<0.01$, ${ }^{* * *} P<$ 0.001. Abbreviations: Neu: control group of untreated neutrophils; Neu+Boc+Leish, Boc-pretreated neutrophils stimulated by $L$. infantum promastigotes; Neu+Leish, untreated neutrophils stimulated by L. infantum promastigotes; Neu+LXA4, untreated neutrophils stimulated by LXA4; Neu+LXA4+Leish, LXA4-pretreated neutrophils stimulated by promastigotes; Neu+Boc+LXA4, Boc-pretreated neutrophils stimulated by LXA4

in neutrophils that are pre-blocked with the lipoxin A4 receptor antagonist Boc and is reversely increased by lipoxin A4 activation before stimulation with promastigotes. Further confirmation is that there was a significant decrease in the proportion of H3Cit-positive neutrophils in lipoxin A4 receptor-silenced HL-60 cells compared with non-silenced neutrophil samples stimulated by promastigotes. The H3Cit expression is consistent with the confocal observations and dsDNA measurement.
Research has demonstrated that the proportion of neutrophils positive for intracellular H3Cit [26] and the presence of plasma H3Cit [27] is correlated with disease severity, and that $\mathrm{H} 3 \mathrm{Cit}$ can serve as a potential biomarker and even therapeutic target of disease [27].

Apart from their ability to regulate neutrophil activation and recruitment, these ligands have a profound influence on neutrophil survival and apoptosis with contrasting effects [19]. Lipoxin A4 is considered as an important anti-inflammatory lipid mediator that is well known as the "stop signal" in inflammatory reactions [28]. Therefore, Leishmania promastigotes may inhibit inflammatory reactions by activating the lipoxin A4 receptor and thereby inducing NETosis. With the establishment of the concept of NET anomaly and exploration of the pathological mechanism of NETs, a better understanding of the signal pathway of NETosis would be of great help to launch interventions. The activation of ERK downstream of PI3K $\gamma$, which occurs via reactive oxygen species-dependent pathways, is associated with $L$. amazonensis-induced NETosis [29]. However, the pathways that are present downstream of Leishmania-induced NET require further exploration. Research has shown that the lipoxin A4 receptor slows down disease progression by inhibiting inflammatory responses and the activation of NF- $\mathrm{KB}$ and/or MAPK pathways [30,31]. With regard to $\mathrm{p} 38$ MAPK and NF- $\mathrm{KB}$ pathways, research has reported that they are required for NET formation [32]. We speculated that the lipoxin A4 receptor may mediate the progression of NETs via NF-kB and ERK/p38 MAPKdependent pathways in $L$. infantum infection. Further experiments to investigate the participation of lipoxin A4 receptor signaling in L. infantum-induced NET formation are needed.

Can NETs effectively resist Leishmania invasion? A study showed that $L$. amazonensis promastigotes were damaged after being captured by NETs, which confirmed the killing effect of NETs in vitro [11]. However, NETs failed to kill L. mexicana in vivo [9]. Based on the DNA skeleton structure of NETs, NETs

\footnotetext{
(See figure on next page.)

Fig. 5 Visualization of NETs by fluorescence staining of DNA, H3Cit and MPO. Purified peripheral blood neutrophils pre-treated with or without the antagonist (Boc) or the agonist (LXA4) were exposed to L. infantum promastigotes for $5 \mathrm{~h}$ at $37^{\circ} \mathrm{C}$ and immunostained for DNA (blue), H3Cit (red) and MPO (green). DNA: DNA backbone is stained with DAPI. H3Cit: citrullinated histone H3 is visualized with a red fluorescence label. MPO: myeloperoxidase is detected with a green fluorescence-labeled specific antibody. Merge: merged image showing fluorescence staining of DNA, H3Cit and MPO. a Control group of untreated neutrophils; b Boc-pretreated neutrophils stimulated by L. infantum promastigotes; $\mathbf{c}$ Untreated neutrophils stimulated by L. infantum promastigotes obviously showing DNA net-like fibers and releasing H3Cit and MPO; $\mathbf{d}$ Untreated neutrophils stimulated by LXA4 expressing H3Cit and MPO; e LXA4-pretreated neutrophils stimulated by promastigotes obviously exhibiting DNA net-like fibers and releasing $\mathrm{H} 3 \mathrm{Cit}$ and $\mathrm{MPO}$; $\mathbf{f}$ Boc-pretreated neutrophils stimulated by LXA4 showing intact cell structure. NETs positive for DNA, H3Cit and MPO were visible in Boc-untreated and lipoxin A4 pre-treated neutrophils stimulated by promastigotes. However, most cells in the Boc-primed neutrophil samples showed intact and smaller nuclei; this means that Boc had an inhibitory effect on NET extrusion via its antagonistic effects on the lipoxin A4 receptor. Scale-bars: $100 \mu \mathrm{m}$
} 


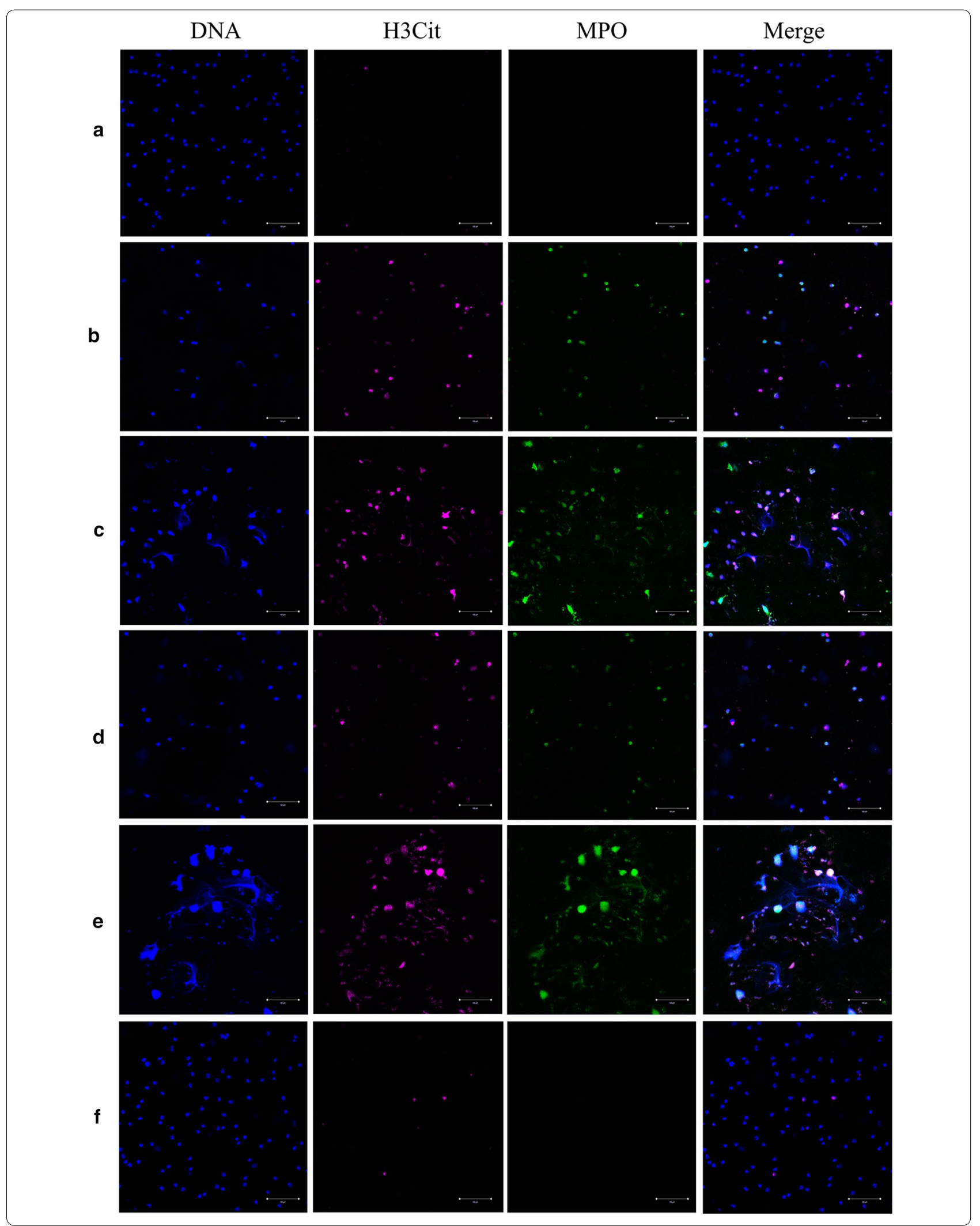


can be degraded by nucleases. The Leishmania membrane anchors a class I nuclease, 3-nucleotidase/nuclease [33], which can destroy the structure of NETs and help the protozoa escape the immunity of NETs [12]. Therefore, whether Leishmania is effectively cleared by NETs is affected by many factors, such as the particular protozoan strain and host characteristics. The desert sub-type strain (MHOM/CN/08/JIASHI-5) involved in this study, which causes the majority of cases $(93.70 \%)$ occurring in the zero- to two-years age group, is highly pathogenic and endemic in the northwestern desert regions of China [34]. Interestingly, comparing the protein expression of $L$. infantum strains from different regions of China by using non-labeled quantitative proteomics, we found that the desert sub-type strain significantly overexpressed 3-nucleotidase/nuclease [35]. Therefore, the desert sub-type strain could effectively be protected against NETs. We speculate that there may be some correlation with the characteristics of the desert sub-type of zoonotic visceral leishmaniasis, but this requires further demonstration.

The overexpression of NETs may have detrimental effects [36], while constitutive activation, dysregulation of suppressive mechanisms and excess NET yield are prominent pathogenic mechanisms that are also likely to contribute to the disease. That is, they could have beneficial or detrimental effects that vary by context. As neutrophils release NETs in response to Leishmania species, it has been proposed that NETs play distinct roles according to the progression of the infection $[37,38]$. The ability of NETs to damage epithelial and endothelial cells and hepatic tissues is well documented $[39,40]$. There is probably an optimal level of NETs that needs to be explored, for which a better understanding of the functions and impact of NETs on health would be useful. DNase treatment was used to abrogate NETmediated chronic inflammation [41]. Further investigation to evaluate the role of the lipoxin A4 receptor in promoting or controlling infection with Leishmania in vivo is the next-step research focus. The lipoxin A4 receptor for neutrophil-mediated immune response to L. infantum infection in our study might offer an alternative target for NET control.

\section{Conclusions}

This study assessed the role of the lipoxin A4 receptor in the neutrophil-mediated immune response to L. infantum infection. A better understanding of the immunological mechanism of NETs in the context of health would be helpful in terms of using this receptor for targeted treatment.

\section{Methods}

\section{Parasite and mice}

The $L$. infantum isolate (MHOM/CN/08/JIASHI-5) was supplied by Dr. Wang (NIPD, China CDC) and was maintained in the promastigote stage at $26{ }^{\circ} \mathrm{C}$ by passage in 199 medium (Lonza Group, Basel, Switzerland) supplemented with heat-inactivated fetal bovine serum (FBS; $30 \mathrm{~min}, 56^{\circ} \mathrm{C}$; Sera Laboratories International, Horsted Keynes, UK) and $100 \mathrm{U} / \mathrm{ml}$ penicillin $+100 \mu \mathrm{g} / \mathrm{ml}$ streptomycin (BioWhittaker, Verviers, Belgium). Parasite concentration was assessed in a Neubauer counting chamber (Paul Marienfeld GmbH \& Co. KG, BM, Germany).

Five-week-old female BALB/c mice (body weight: $16 \pm$ $2 \mathrm{~g}$ ) were purchased from Shanghai Jihui Laboratory Animal Feeding Co. Ltd. The mice were adaptively fed with standard laboratory chow and sacrificed for research. All experimental procedures were approved by the Laboratory Animals Welfare and Ethics Committee of National Institute of Parasitic Diseases, Chinese Center for Diseases Control and Prevention.

\section{In vitro $L$. infantum infection and flow cytometric analysis of whole blood}

Whole blood samples were obtained through the retroorbital bleeding method and collected in tubes containing heparin and used within $1 \mathrm{~h}$. A $0.2-\mathrm{ml}$ volume of heparin-containing blood from each mouse was seeded in a 24-well plate containing $L$. infantum promastigotes at a parasite:neutrophil ratio of 10:1 in $300 \mu \mathrm{l}$ of RPMI 1640 medium supplemented with $10 \%$ heat-inactivated FBS (v/v) for $5 \mathrm{~h}$. There were six experimental groups: whole blood stimulated by promastigotes without pretreatment, whole blood pre-incubated for $30 \mathrm{~min}$ with $10 \mu \mathrm{M}$ Boc prior to stimulation with promastigotes or 10 $\mathrm{ng} / \mathrm{ml}$ LXA4 (an endogenous lipid mediator/activator of the lipoxin A4 receptor), whole blood pre-treated with 10 $\mathrm{ng} / \mathrm{ml}$ LXA4 with or without promastigote stimulation later, and whole blood without any treatment was used as the control group. Three independent experiments were performed. The NETs were quantified by flow cytometric assay.

\section{Isolation of neutrophils from peripheral blood}

Whole blood samples from mice were obtained through the retro-orbital bleeding method, collected in tubes containing heparin and used within $1 \mathrm{~h}$. Neutrophil isolation was achieved using mouse peripheral blood neutrophil purification kit (TBD, Nanjing, China). Isolated cells were suspended in RPIM 1640 supplemented with 10\% heat-inactivated FBS (v/v) (Gibco, San Diego, CA, USA), and their viability and concentration were assessed by trypan blue exclusion in a Neubauer counting chamber before any of the assays were performed. Cell purity was 
determined by flow cytometry analysis and microscopic observation. Neutrophil purity was confirmed to be > $85 \%$ by immunostaining with CD11b and Ly6G.

\section{In vitro stimulation of purified neutrophils with $L$. infantum promastigotes}

Purified neutrophils (final concentration, $2 \times 10^{5}$ cells/ well) were seeded in 24-well plates (Nunc, Roskilde, Denmark) with $L$. infantum promastigotes at a parasiteneutrophil ratio of 10:1 in RIPM 1640 supplemented with $10 \%$ heat-inactivated FBS $(\mathrm{v} / \mathrm{v})$. Plates were incubated at $37{ }^{\circ} \mathrm{C}$ in a humidified atmosphere containing $5 \%$ of $\mathrm{CO}_{2}$ for $5 \mathrm{~h}$ to allow the induction of NETs. The experimental groups were the same as described earlier. The neutrophil cultures were used in the following experiments: measurement of extracellular dsDNA and microscopic assessment of NETs.

\section{HL-60 cell culture}

The human acute promyelocytic leukemia cell line HL-60 (provided by the Cell Bank of the Chinese Academy of Sciences) was maintained in RIPM 1640 medium supplemented with $10 \%$ heat-inactivated FBS $(\mathrm{v} / \mathrm{v})$ in $5 \%$ $\mathrm{CO}_{2}$ at $37^{\circ} \mathrm{C}$. Cells were seeded at a density of $5 \times 10^{5}$ cells $/ \mathrm{ml}$ and cultured for 5 days in the above-mentioned cell medium supplemented with $1.25 \%$ DMSO to induce their differentiation into neutrophil-like cells [21]. Fresh medium was added on the third day of culture to prevent cell overgrowth and depletion of nutrients.

\section{Lipoxin A4 receptor gene silencing}

HL-60 cells stimulated to undergo neutrophil-like differentiation were seeded in 24-well plates (final concentration, $2 \times 10^{5}$ cells/well). On day 3 of differentiation, the cells were transfected with control or specific siRNA against the lipoxin A4 receptor [42] by using Lipofectamine $^{\text {TM }}$ RNAiMAX (Thermo Fisher Scientific, Waltham, MA, USA) for $48 \mathrm{~h}$ Then, $3 \mu \mathrm{l}$ of the transfection reagent and $50 \mathrm{nM}$ siRNA were diluted separately in $50 \mu \mathrm{l}$ of Opti-MEM (Gibco) and incubated for $10 \mathrm{~min}$ at room temperature. The above solutions were mixed and incubated for a further $5 \mathrm{~min}$. Subsequently, the Lipofectamine-siRNA complexes $(50 \mu \mathrm{l})$ were added to the culture medium and the cells were incubated for $48 \mathrm{~h}$ for further analysis.

\section{Quantitative reverse transcriptase-polymerase chain reaction}

The total RNA of HL-60 cells was extracted with Trizol reagent (Invitrogen, Carlsbad, CA, USA) and reverse-transcribed using a cDNA reverse transcription kit (TaKaRa, Dalian, Japan). To determine the efficiency of lipoxin A4 receptor gene down-regulation, the reverse-transcribed cDNA was used as a template in qPCR reaction mixtures containing SYBR Green Realtime PCR Master Mix (TaKaRa) and $0.4 \mu \mathrm{M}$ forward and reverse primers. The following primers were utilized: GAPDH forward [21] (5'-CCC CTT CAT TGA CCT CAA CTA C-3'), GAPDH reverse (5'-GAG TCC TTC CAC GAT ACC AAA G-3'); lipoxin A4 receptor forward [43] (5'-GTG ATC TGG GTG GCT GGA TT-3'); and lipoxin A4 receptor reverse (5'-AGG GCC AGG TTC AGG TAA CA- $3^{\prime}$ ). Data are shown as relative mRNA levels normalized to GAPDH.

\section{In vitro stimulation of differentiated HL-60 cells with $L$. infantum promastigotes}

Differentiated HL-60 cells with or without lipoxin A4 receptor gene silencing (final concentration, $2 \times 10^{5}$ cells/ well) were seeded in 24-well plates (Nunc) with $L$. infantum promastigotes at a parasite-cell ratio of 10:1 in RIPM 1640 medium supplemented with $10 \%$ heat-inactivated FBS (v/v). Plates were incubated at $37^{\circ} \mathrm{C}$ in a humidified atmosphere containing $5 \%$ of $\mathrm{CO}_{2}$ for $5 \mathrm{~h}$ to allow the induction of NETs. Differentiated HL-60 cells without any treatment was used as the control group. Three independent experiments were performed. NET quantification was performed with flow cytometric analysis.

\section{Flow cytometric analysis of NETs}

The in vitro infected samples were collected into tubes and centrifuged at $1000 \times g$ for $10 \mathrm{~min}$. The precipitate was incubated with $2 \mu \mathrm{l}$ of rabbit polyclonal anti-Histone $\mathrm{H} 3$ antibody (citrulline R2 + R8 + R17) (Abcam, Cambridge, UK) for $30 \mathrm{~min}$ at room temperature. Following this, $1 \mu \mathrm{l}$ of anti-CD11b-PE (eBioscience, San Diego, CA, USA) and anti-Ly6G-BV421 (eBioscience) antibodies, and $0.8 \mu \mathrm{l}$ of AlexaFluor-700-conjugated goat antirabbit antibody (Thermo Scientific, Rockford, IL, USA) were added and incubated for $30 \mathrm{~min}$ at room temperature in the dark. Then, for whole blood samples, $1 \mathrm{ml}$ of FACS reagent (BD Biosciences, San Diego, CA, USA) was added until red cell lysis was achieved (10-15 min). This was followed by the addition of $1 \mathrm{ml}$ of $2 \%$ bovine serum albumin in phosphate-buffered saline (PBS) and centrifugation at $1000 \times g$ for $10 \mathrm{~min}$. The supernatant was discarded, and the pellet was resuspended in $300 \mu$ of PBS. Labeled cells were kept on ice and examined with flow cytometry analysis (FACS LX, Beckman-Coulter, Pasadema, CA, USA), which was performed with the CytExpert software (Beckman). The percentage of isotype controls was $<3 \%$. At least 10,000 neutrophil or neutrophil-like populations were collected for each sample. Three independent experiments were performed. 


\section{Measurement of extracellular dsDNA}

NETs were quantified in the culture supernatant with the Quant-iT ${ }^{\mathrm{TM}}$ PicoGreen ${ }^{\circledR}$ dsDNA Assay kit (Thermo Fisher Scientific) according to the manufacturer's instructions. Samples were distributed into 96-well plates and read in a spectrofluorometer (SpectraMax M5; Molecular Devices, Sunnyvale, CA, USA) with a filter setting of $480 \mathrm{~nm}$ (excitation) and $520 \mathrm{~nm}$ (emission).

\section{Microscopic assessment of NETs}

Fluorescence microscopy and scanning electron microscopy were used for the visualization of NETs. For fluorescence microscopy, slides with adherent neutrophils were fixed with $4 \%$ paraformaldehyde (SigmaAldrich, Santa Clara, CA, USA) for $20 \mathrm{~min}$, blocked with mouse serum, stained with rabbit polyclonal anti-Histone $\mathrm{H} 3$ antibody (citrulline $\mathrm{R} 2+\mathrm{R} 8+\mathrm{R} 17$ ) (Abcam) at $4{ }^{\circ} \mathrm{C}$ overnight, and washed with PBS three times. This was followed by staining with AlexaFluor700-conjugated goat anti-rabbit antibody (Thermo Scientific) and anti-MPO-FITC antibody (Abcam) for 60 min each. Finally, DAPI (4,6-diamidino-2-phenylindole; Sigma-Aldrich) staining was performed for $10 \mathrm{~min}$. All specimens were observed, and photographs of the relevant fields were taken under a confocal microscope $(20 \times$ objective, $10 \times$ eyepiece; ECLIPSE-TI, Nikon, Tokyo, Japan).

For scanning electron microscopy, the coverslips seeded with samples were washed with PBS, fixed with $1 \mathrm{ml}$ Monti-Graziadei solution, and processed for scanning electron microscopy.

\section{Statistical analysis}

Data were expressed as mean \pm standard deviation (SD) of triplicate wells in three independent experiments. All statistical analyses were performed using SPSS version 20.0. Differences based on the data characteristics were analyzed by one-way ANOVA and Student's t-test. All graphs were performed with the GraphPad Prism 7 software. $P$-values $<0.05$ were considered to indicate statistical significance.

\section{Abbreviations}

NETs: neutrophil extracellular traps; Boc: N-t-Butoxycarbonyl-phenylalanineleucyl-phenylalanine-leucyl-phenylalanine; LXA4: lipoxin A4; MPO: myeloperoxidase; $\mathrm{H} 3 \mathrm{C}$ it: citrullinated histone $\mathrm{H} 3$.

\section{Acknowledgements}

The authors are grateful to Jian Guo and Feng Shi of the National Institute of Parasitic Diseases, Chinese Center for Disease Control and Prevention, China, for their help with the confocal immunofluorescence microscopy observations and figures processing.

\section{Authors' contributions}

FW, WG, JW, YW and JC conceived and designed the experiments. FW, YY and $J \mathrm{~L}$ performed the experiments. FW analyzed the data and wrote the paper. JC revised the paper. All authors read and approved the final manuscript.

\section{Funding}

This study was supported by the National Natural Science Foundation of China (no. 81772225 to JC), the Chinese Special Program for Scientific Research of Public Health (no. 201502021 to JC) and the Fourth Round of Three-Year Public Health Action Plan of Shanghai, China (no. 15GWZK0101 to JC). The funders had no role in the study design, data collection and analysis, the decision to publish, or preparation of the manuscript.

\section{Availability of data and materials}

The datasets supporting the conclusions of this article are included within the article.

\section{Ethics approval and consent to participate}

This study was carried out in strict accordance with the recommendations of the Guide for the Care and Use of Laboratory Animals of the Ministry of Science and Technology, China. The protocol was approved by the Laboratory Animals Welfare and Ethics Committee of National Institute of Parasitic Diseases, Chinese Center for Diseases Control and Prevention (Permit Number: IPD-2014-2). All the animal carcasses were disposed of by the Shanghai Animal Harmless Treatment Center. A special effort was made to reduce the number of mice used in the study, to provide them with the most comfortable conditions, and to minimize animal suffering where possible.

\section{Consent for publication}

Not applicable.

\section{Competing interests}

The authors declare that they have no competing interests.

\section{Author details}

${ }^{1}$ National Institute of Parasitic Diseases, Chinese Center for Disease Control and Prevention, Shanghai 200025, China. ${ }^{2}$ Chinese Center for Tropical Diseases Research, Shanghai 200025, China. ${ }^{3}$ WHO Collaborating Center for Tropical Diseases, Shanghai 200025, China. ${ }^{4}$ National Center for International Research on Tropical Diseases, Ministry of Science and Technology, Shanghai 200025, China. ${ }^{5}$ Key Laboratory of Parasite and Vector Biology, Ministry of Health, Shanghai 200025, China.

Received: 20 November 2018 Accepted: 23 May 2019

Published online: 29 May 2019

\section{References}

1. Scott P. Leishmania - a parasitized parasite. N Engl J Med. 2011;364:1773-4

2. Faria MS, Reis FC, Lima AP. Toll-like receptors in Leishmania infections: guardians or promoters? J Parasitol Res. 2012;2012:930257.

3. Carlsen ED, Liang Y, Shelite TR, Walker DH, Melby PC, Soong L. Permissive and protective roles for neutrophils in leishmaniasis. Clin Exp Immunol. 2015;182:109-18.

4. Hurrell BP, Regli IB, Tacchini-Cottier F. Different Leishmania species drive distinct neutrophil functions. Trends Parasitol. 2016;32:392-401.

5. Pompeu ML, Freitas LA, Santos ML, Khouri M, Barral-Netto M. Granulocytes in the inflammatory process of BALB/C mice infected by Leishmania amazonensis. A quantitative approach. Acta Trop. 1991;48:185-93.

6. Matte C, Olivier M. Leishmania-induced cellular recruitment during the early inflammatory response: modulation of proinflammatory mediators. J Infect Dis. 2002;185:673-81.

7. Thalhofer CJ, Chen Y, Sudan B, Love-Homan L, Wilson ME. Leukocytes infiltrate the skin and draining lymph nodes in response to the protozoan Leishmania infantum chagasi. Infect Immun. 2011;79:108-17.

8. Ribeiro-Gomes FL, Sacks D. The influence of early neutrophil-Leishmania interactions on the host immune response to infection. Front Cell Infect Microbiol. 2012;2:59. 
9. Hurrell BP, Schuster S, Grun E, Coutaz M, Williams RA, Held W, et al. Rapid sequestration of Leishmania mexicana by neutrophils contributes to the development of chronic lesion. PLoS Pathog. 2015;11:e1004929.

10. Papayannopoulos V. Neutrophil extracellular traps in immunity and disease. Nat Rev Immunol. 2018;18:134-47.

11. Guimaraes-Costa AB, Nascimento MT, Froment GS, Soares RP, Morgado FN, Conceicao-Silva F, et al. Leishmania amazonensis promastigotes induce and are killed by neutrophil extracellular traps. Proc Natl Acad Sci U S A. 2009:106:6748-53.

12. Guimaraes-Costa AB, DeSouza-Vieira TS, Paletta-Silva R, Freitas-Mesquita AL, Meyer-Fernandes JR, Saraiva EM. 3'-nucleotidase/nuclease activity allows Leishmania parasites to escape killing by neutrophil extracellular traps. Infect Immun. 2014;82:1732-40.

13. Gabriel C, McMaster WR, Girard D, Descoteaux A. Leishmania donovani promastigotes evade the antimicrobial activity of neutrophil extracellular traps. J Immunol. 2010;185:4319-27.

14. van Zandbergen $\mathrm{G}$, Hermann N, Laufs H, Solbach W, Laskay T. Leishmania promastigotes release a granulocyte chemotactic factor and induce interleukin-8 release but inhibit gamma interferon-inducible protein 10 production by neutrophil granulocytes. Infect Immun. 2002;70:4177-84.

15. Fredman G, Ozcan L, Spolitu S, Hellmann J, Spite M, Backs J, et al. Resolvin D1 limits 5-lipoxygenase nuclear localization and leukotriene B4 synthesis by inhibiting a calcium-activated kinase pathway. Proc Natl Acad Sci U S A. 2014;111:14530-5.

16. Wu R, Zhou W, Chen S, Shi Y, Su L, Zhu M, et al. Lipoxin A4 suppresses the development of endometriosis in an ALX receptor-dependent manner via the p38 MAPK pathway. Br J Pharmacol. 2014;171:4927-40.

17. $\mathrm{Xu} Z$, Zhao F, Lin $F$, Xiang $H$, Wang $N$, Ye D, et al. Preeclampsia is associated with a deficiency of lipoxin $A 4$, an endogenous anti-inflammatory mediator. Fertil Steril. 2014;102(282-90):e4.

18. Li L, Chen K, Xiang Y, Yoshimura T, Su S, Zhu J, et al. New development in studies of formyl-peptide receptors: critical roles in host defense. J Leukoc Biol. 2015;99:425-35.

19. El Kebir D, József L, Filep JG. Opposing regulation of neutrophil apoptosis through the formyl peptide receptor-like 1/lipoxin A4 receptor: implications for resolution of inflammation. J Leukoc Biol. 2008;84:600-6.

20. Wenzel A, Van Zandbergen G. Lipoxin A4 receptor dependent Leishmania infection. Autoimmunity. 2009:42:331-3.

21. Khandagale A, Lazzaretto B, Carlsson G, Sundin M, Shafeeg S, Romling $\mathrm{U}$, et al. JAGN1 is required for fungal killing in neutrophil extracellular traps: implications for severe congenital neutropenia. J Leukoc Biol. 2018:104:1199-213.

22. Wang Y, Li M, Stadler S, Correll S, Li P, Wang D, et al. Histone hypercitrullination mediates chromatin decondensation and neutrophil extracellular trap formation. J Cell Biol. 2009;184:205-13.

23. Li P, Li M, Lindberg MR, Kennett MJ, Xiong N, Wang Y. PAD4 is essential for antibacterial innate immunity mediated by neutrophil extracellular traps. J Exp Med. 2010;207:1853-62.

24. Thålin C, Demers M, Blomgren B, Wong SL, Von AM, Von HA, et al. NETosis promotes cancer-associated arterial microthrombosis presenting as ischemic stroke with troponin elevation. Thromb Res. 2016:139:56.

25. Lee KH, Cavanaugh L, Leung H, Yan F, Ahmadi Z, Chong BH, et al. Quantification of NETs-associated markers by flow cytometry and serum assays in patients with thrombosis and sepsis. Int J Lab Hematol. 2018;40:392-9.

26. Thålin C, Lundström S, Seignez C, Daleskog M, Lundström A, Henriksson $\mathrm{P}$, et al. Citrullinated histone $\mathrm{H} 3$ as a novel prognostic blood marker in patients with advanced cancer. PLoS ONE. 2018;13:e0191231.

27. Li Y, Liu Z, Liu B, Zhao T, Chong W, Wang Y, et al. Citrullinated histone H3: a novel target for the treatment of sepsis. Surgery. 2014;156:229-34.
28. Guo YP, Jiang HK, Jiang H, Tian HY, Li L. Lipoxin A4 may attenuate the progression of obesity-related glomerulopathy by inhibiting NF-KB and ERK/p38 MAPK-dependent inflammation. Life Sci. 2018;198:112-8.

29. DeSouza-Vieira T, Guimarães-Costa A, Rochael NC, Lira MN, Nascimento MT, Lima-Gomez PS, et al. Neutrophil extracellular traps release induced by Leishmania: role of PI3KY, ERK, PI3Ko, PKC, and [Ca2+]. J Leukoc Biol. 2016;100:801-10.

30. Luo CL, Li QQ, Chen XP, Zhang XM, Li LL, Li BX, et al. Lipoxin A4 attenuates brain damage and downregulates the production of pro-inflammatory cytokines and phosphorylated mitogen-activated protein kinases in a mouse model of traumatic brain injury. Brain Res. 2013;1502:1-10.

31. Wang YP, Wu Y, Li LY, Zheng J, Liu RG, Zhou JP, et al. Aspirin-triggered lipoxin $\mathrm{A} 4$ attenuates LPS-induced pro-inflammatory responses by inhibiting activation of NF-KB and MAPKs in BV-2 microglial cells. J Neuroinflammation. 2011;8:95.

32. Tatsiy O, Mcdonald PP. Physiological stimuli induce PAD4-dependent, ROS-independent NETosis, with early and late events controlled by discrete signaling pathways. Front Immunol. 2018:9:2036.

33. Joshi MB, Dwyer DM. Molecular and functional analyses of a novel class I secretory nuclease from the human pathogen, Leishmania donovani. J Biol Chem. 2007;282:10079-95.

34. Wang JY, Cui G, Chen HT, Zhou XN, Gao CH, Yang YT. Current epidemiological profile and features of visceral leishmaniasis in people's republic of China. Parasit Vectors. 2012;5:31

35. Wei FR, Gao CH, Wang JY, Yang YT, Shi F. Comparative proteomics analysis of Leishmania infantum strains isolated from two epidemiological regions of visceral leishmaniasis in China. Chin J Schisto Control. 2018;30:625-9.

36. Kapoor S, Opneja A, Nayak L. The role of neutrophils in thrombosis. Thromb Res. 2018;170:87-96.

37. Gardinassi LG, DeSouza-Vieira TS, da Silva NO, Garcia GR, Borges VM, Campos RNS, et al. Molecular signatures of neutrophil extracellular traps in human visceral leishmaniasis. Parasit Vectors. 2017;10:285.

38. Morgado FN, Nascimento MT, Saraiva EM, De OR, Madeira FM, Da CS, et al. Are neutrophil extracellular traps playing a role in the parasite control in active american tegumentary leishmaniasis lesions? PLOS ONE. 2015;10:e0133063.

39. Saffarzadeh M, Juenemann C, Queisser MA, Lochnit G, Barreto G, Galuska SP, et al. Neutrophil extracellular traps directly induce epithelial and endothelial cell death: a predominant role of histones. PLOS ONE. 2012; 7:e32366.

40. Thomas GM, Carbo C, Curtis BR, Martinod K, Mazo IB, Schatzberg D, et al. Extracellular DNA traps are associated with the pathogenesis of TRALI in humans and mice. Blood. 2012;119:6335-43.

41. Warnatsch A, loannou M, Wang Q, Papayannopoulos V. Inflammation. Neutrophil extracellular traps license macrophages for cytokine production in atherosclerosis. Science. 2015;349:316-20.

42. Ramon S, Bancos S, Serhan CN, Phipps RP. Lipoxin A4 decreases human memory B cell antibody production via an ALX/FPR2-dependent mechanism: a link between resolution signals and adaptive immunity. Eur J Immunol. 2014;44:357.

43. Babu PS. A truncated form of CKbeta8-1 is a potent agonist for human formyl peptide-receptor-like 1 receptor. Br J Pharmacol. 2010;141:37-46.

\section{Publisher's Note}

Springer Nature remains neutral with regard to jurisdictional claims in published maps and institutional affiliations. 